\title{
BACKING UP A TrAILER - AN APPLICATION-BASED VeCtOR ANALYSIS ProJect FOR 2ND Year CALCUluS
}

\author{
Peter Teertstra and Sanjeev Bedi \\ Department of Mechanical and Mechatronics Engineering \\ University of Waterloo \\ peter.teertstra@uwaterloo.ca
}

\begin{abstract}
A project for the second year calculus course, ME201, will be presented. Students worked in groups, make measurements using physical prototypes and performed analysis to predict behavior for the physical situation of reversing (backing up) a vehicle attached to a single axle trailer. The objectives of the project were to reinforce concepts from previous courses, including physics (vector components) and linear algebra (matrices), to demonstrate key concepts from the current course (vector products, moment vector), and to generate interest in future courses (sensors and feedback, control systems). This paper will outline the development process for the project, including the design and fabrication of the physical prototypes and the selection of test conditions. Measurements and analysis from the project, including samples of student work, will be presented.
\end{abstract}

Keywords: Vector calculus, vector products, undergraduate project

\section{INTRODUCTION}

ME201 Advanced Calculus, the third calculus course taken by Mechanical Engineering students at University of Waterloo, has topics that include integration and differentiation of multivariable functions, vectors and vector calculus for single and multivariable functions, and line and surface integrals of scalar and vector functions. The author has taught this course for more than a decade and has often struggled to find a project that is engaging and relevant to students as well as supportive of the course material.

An idea for an application-based project involving the physical situation of reversing (backing up) a vehicle attached to a single axle trailer was provided by Sanjeev Bedi, a faculty member in the Department of Mechanical and Mechatronics Engineering at Waterloo and the NSERC Chair, Engineering IDEAs Clinic, a faculty-wide resource to assist programs and instructors develop and deploy open-ended learning activities for their students. The objectives of the project were to reinforce concepts from previous courses, including physics (vector components) and linear algebra (matrices), to demonstrate key concepts from the current course (vector products, moment vector), and to generate interest in future courses (sensors and feedback, control systems).

This paper will outline the development process for the project, including designing and fabricating the physical prototypes, establishing test conditions, and developing learning materials to review key course concepts. Samples of student work will be provided and a number of recommendations will be presented for future implementations of the project.

\section{MATHEMATICAL MODEL}

The mathematical model used to simulate the position of a trailer relative to a vehicle as it is being backed up is based on the geometry and relative position of each through the use of the instantaneous center. The instantaneous center is the point at the center of the circular paths travelled by each of the vehicle wheels as the car turns at a fixed steering angle. For the vehicle, the instantaneous center is located at the intersection of lines drawn perpendicular to each of the four wheels, as shown in Fig. 1. In the case of the trailer, the instantaneous center is located at the intersection of lines drawn parallel to the axle and perpendicular to the force applied at the connection point to the vehicle (the hitch), as shown in Fig. 2.

The objective of the model, to predict the path of the trailer relative to the vehicle as a function of steering wheel position, is achieved using the scalar and vector products of the geometry from the instantaneous centers, as follows.

STEP 1 The vector representing the force applied by the vehicle at the center steering wheel position to the trailer is formed which is normal to a line connecting the instantaneous center of the vehicle and the vehicle hitch point (see Fig. 1). 


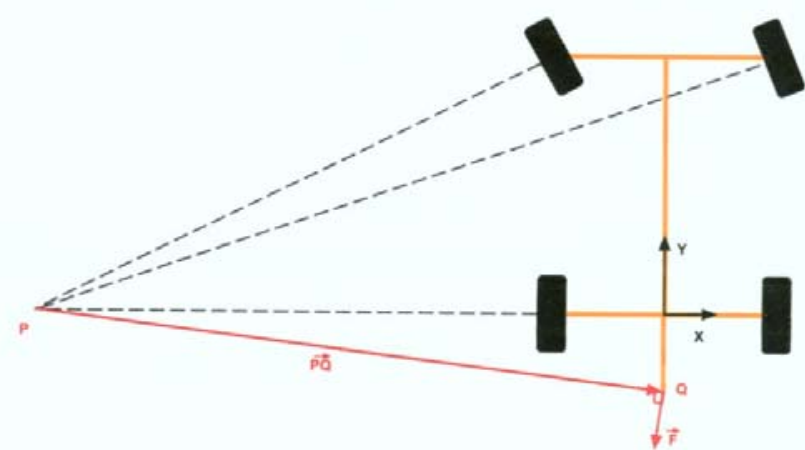

Fig. 1. Instantaneous Center for Vehicle [4].

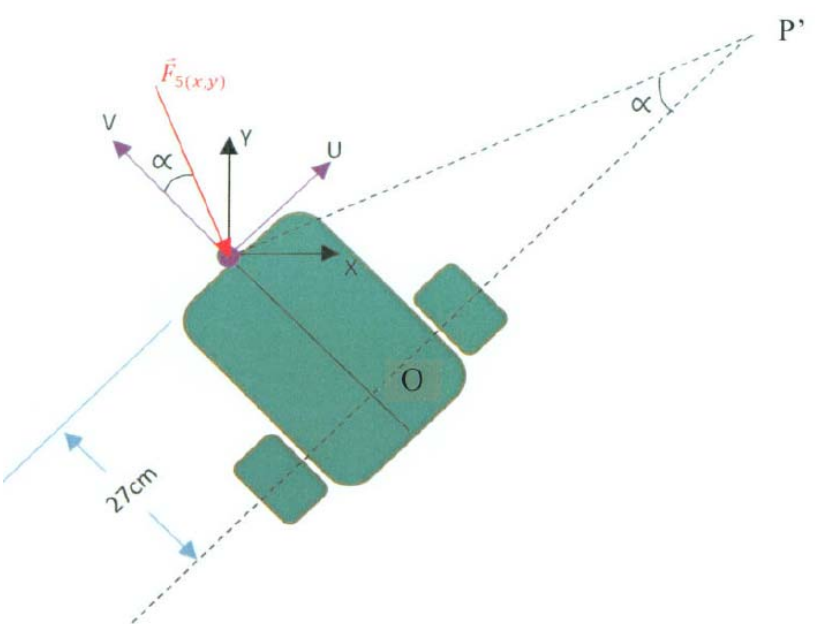

Fig. 2. Instantaneous Center for Trailer [2].

STEP 2 A rotational matrix is used to relate the reference coordinate system $(x, y)$ for the vehicle to the rotated coordinate system $(u, v)$ for the trailer, according to the angle at the hitch, $\theta$, where the $\mathrm{u}$-axis and v-axis

$$
\left\{\begin{array}{c}
F_{u} \\
F_{v}
\end{array}\right\}=\left[\begin{array}{cc}
\cos \theta & \sin \theta \\
-\sin \theta & \cos \theta
\end{array}\right]\left\{\begin{array}{l}
F_{x} \\
F_{y}
\end{array}\right\}
$$

of the coordinate system are aligned with the trailer axle and centerline, respectively, and the origin is placed at the trailer hitch point, as shown in Fig. 2.

STEP 3 The force exerted by the vehicle on the trailer is used to form a perpendicular line which intersects a line parallel to the trailer axle at the instantaneous center, as shown in Fig. 2.

STEP 4 The instantaneous center of the trailer is used to trace circular paths at each of the trailer wheels that predict their motion relative to the vehicle. This path is followed for a short time until the angle $\theta$ changes substantially.

\section{APPARATUS AND PROCEDURE}

The development of this project was performed in the four months of the Spring, 2016 academic term, including the physical prototypes, project description and the learning materials to review key course concepts.

\subsection{Physical Prototypes}

The physical prototypes were constructed with the assistance of the Engineering Machine Shop at the University of Waterloo and the Mechanical and Mechatronics Engineering Clinic (WATiMake), as follows.

Six trailer prototypes were constructed as shown in Fig. 3. The trailers were approximately $30 \mathrm{~cm}$ long by $23 \mathrm{~cm}$ wide, and each included two different hitch styles; one that included a force sensor and was connected to a push rod, and one that was compatible with the vehicle hitch. The trailer included an area where weights could be added as required by the test procedure.

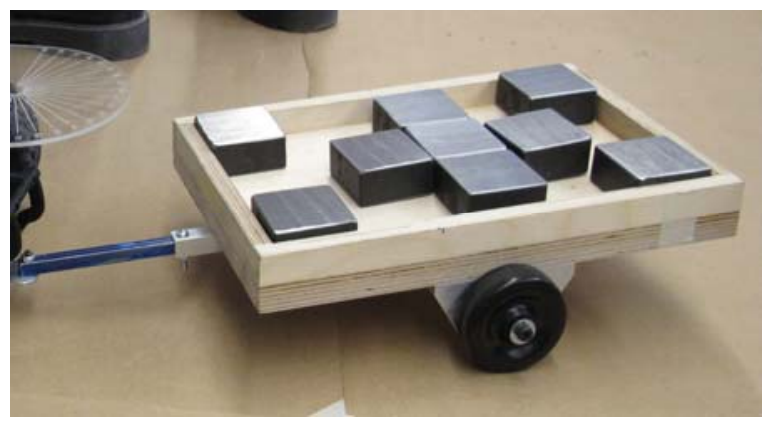

Fig. 3. Trailer Prototype.

The vehicle prototypes used by the students were Traxxas Telluride radio controlled (RC) cars that were modified specifically for this project, as shown in Fig. 4.

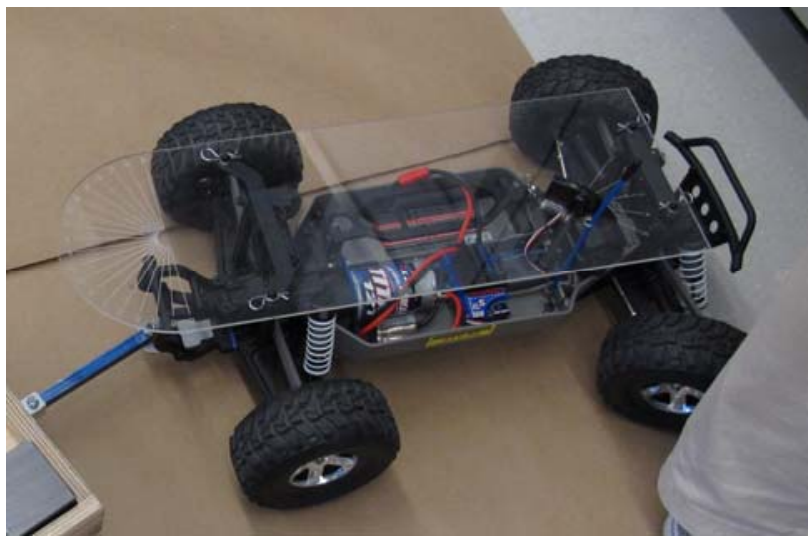

Fig. 4. Vehicle Prototype. 
The steering servo was disconnected and replaced with a simple manual knob. The pointer / indicator system allowed for 5 pre-set steering positions, corresponding to straight ahead and instantaneous center locations of 55 $\mathrm{cm}$ and $110 \mathrm{~cm}$ to the left and right of the vehicle, and these instantaneous centers were provided to the students.

\subsection{Project Description and Procedure}

The students were provided with a detailed project description during Week 9 of the term immediately following the midterm exam. The timing of the project introduction was selected to correspond with the conclusion of a series of lectures on the applications of the vector products, including the calculation of moment vectors based on the cross product and scalar components of vectors based on the dot product.

Students worked in groups of 4 to perform all measurements, analysis and reporting for the project. The students were directed to work through the project in 3 distinct steps. Here are the instructions that the students followed for each part of the project.

\section{Part 1 - Backing Up a Trailer By Hand}

These measurements used the trailer prototype equipped with a push rod and force sensor, $4.5 \mathrm{~kg}$ of weights, a large sheet of kraft paper $(60 \times 150 \mathrm{~cm})$, a protractor and a tape measure.

A long straight line at least 2 meters long was made using masking tape in an open area on the floor and the trailer and push rod were placed in line with each other, overtop of the taped line at one end. The weights were placed in the trailer and the trailer was slowly moved backwards at a constant speed using the push rod, keeping the push rod aligned with the line on the paper at all times. As the trailer was pushed the force required to push the trailer as a function of distance travelled along the line, and the path of trailer wheels relative to the straight line were both recorded. Force data and the paths of the wheels were recorded until the trailer "jackknifed," that is when the angle between the pushing force and the trailer reached 90 degrees.

The trailer was then positioned at an appropriate starting point on a large piece of kraft paper taped to the floor in an open area. The push rod was set to an angle of $120^{\circ}$ relative to the trailer hitch and the trailer was slowly pushed at a constant speed using the push rod, while the angle between the push rod and the trailer was kept constant. The force required to push the trailer as a function of distance travelled was recorded and the path of trailer wheels was traced on the paper. The

measurements were continued until the trailer wheels left the paper. This procedure was then repeated for an angle of $150^{\circ}$ between the push rod and the trailer hitch.

\section{Part 2 - Analysis}

The analysis was performed in two parts. In the first part, the results of the force measurements were collected and plotted for the three cases; where the angle between the force vector and the trailer was held at $120^{\circ}, 150^{\circ}$ and where the angle varied between $180^{\circ}$ and $90^{\circ}$ (when the trailer "jack knifed.")

The instantaneous center was also calculated for each of the cases where the angle was held constant. From this the direction and magnitude of the vector from the instantaneous center to the trailer hitch, $\overrightarrow{P Q}$, was found.

(The hand drawn sketch shown in Fig. 5 was included in the project description.) Next it was verified that vector

$\overrightarrow{P Q}$ was normal to the force vector and the moment vector was calculated for this geometry.

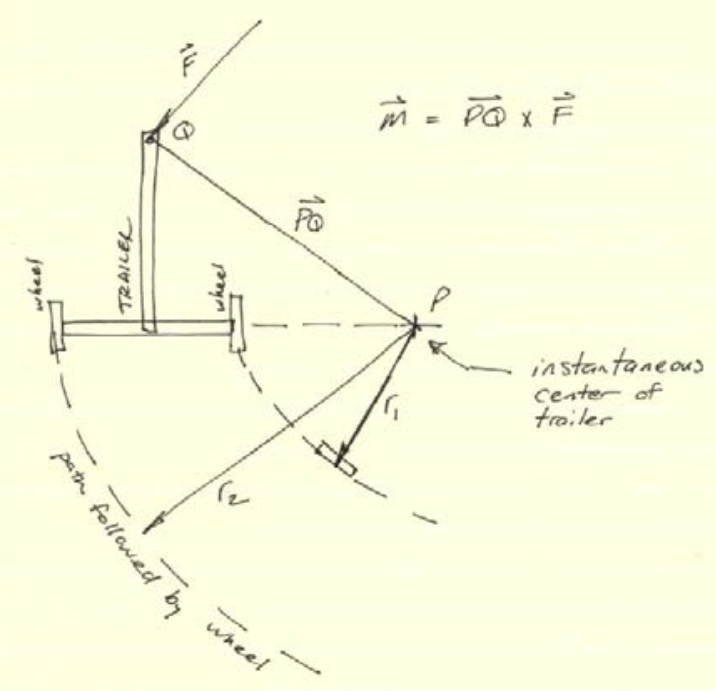

Fig. 5. Schematic Provided to Demonstrate Instantaneous Center and Vector Product for Trailer.

In the second part of the analysis, the path followed by the trailer when it is connected to the vehicle was predicted for 5 different cases. The hand drawn sketch shown in Fig. 6 was included in the project description. In each case, the mathematical model described in Section 2 based on vector products from the instantaneous centers and the rotational matrix was used for the calculations

\section{Part 3 - Backing Up a Trailer Using a Vehicle}

These measurements used the vehicle and trailer prototypes, two or three large sheet of kraft paper $(60 \mathrm{x}$ $150 \mathrm{~cm}$ ), a marker, string, a protractor and a tape measure.

A large piece of kraft paper was taped to an open area on the floor and the vehicle and trailer were placed at an appropriate position for Case \#1. The calculated instantaneous center for the trailer for Case \#1 was marked on the paper and the string and a marker were used to trace the predicted path of the trailer wheels. The 


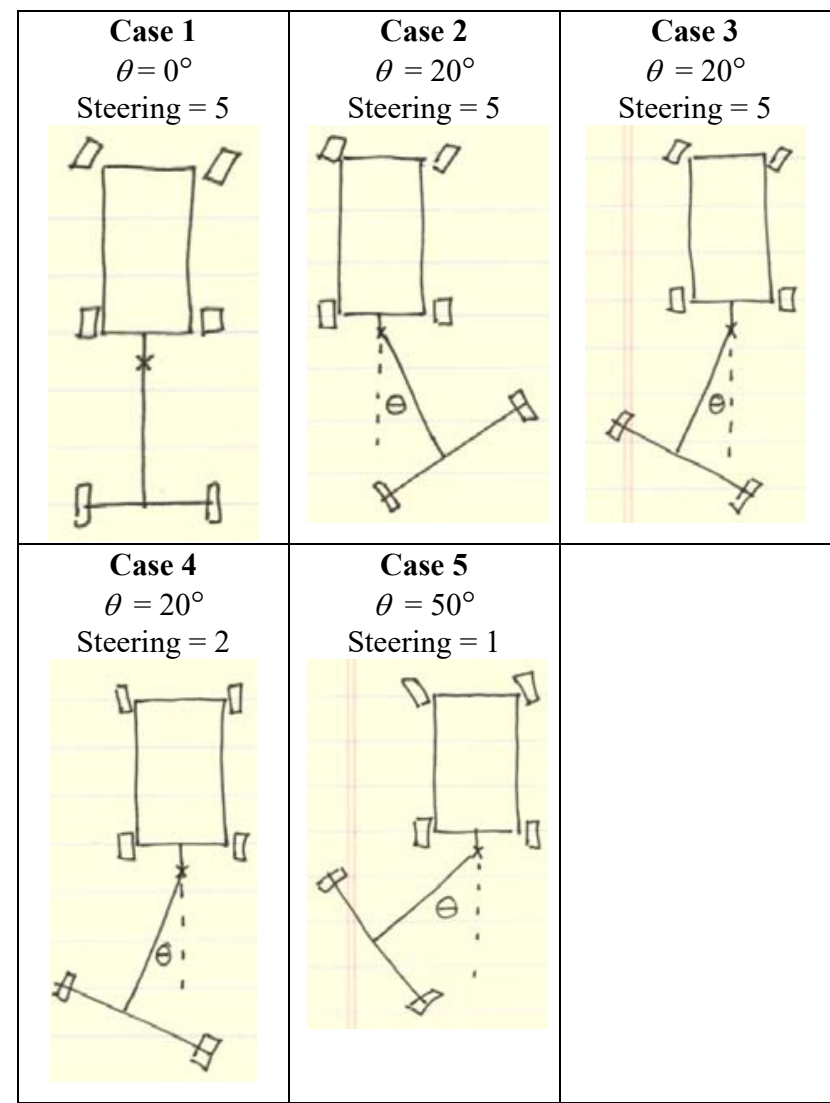

Fig. 6. Schematics Provided to Describe Five Analysis Cases.

vehicle and trailer were slowly pushed backwards and the path of each of the trailer wheels was drawn on the kraft paper using the marker. This was continued until the predicted and actual paths diverged significantly. The same procedure was repeated for Cases \#2, 3, 4 and 5.

\subsection{Learning Materials}

At the introduction of the project to the class, a special 50 minute lecture was presented to reinforce key concepts and provide specific information regarding the analysis. The following is an outline of the key elements in that lecture.

The presentation began with the motivation for the project, as follows: "Many drivers use a trial-and-error approach when they back up a vehicle with a trailer attached: 'When I turn the steering wheel this way, this happens... and when I turn it the other way, something else happens" "This was demonstrated to the class using a public-domain, internet based video showing an individual struggling to back up a travel trailer.

The class was then told that "the physical laws that govern how a trailer moves relative to a vehicle can be modelled based on the geometry and relative position of each" and that "this model can be implemented into a control system that can assist drivers in backing up." This was demonstrated to the class using an advertisement for a manufacturer of transport trucks featuring a steering system that aids drivers in backing up.

The first series of slides presented the concept of instantaneous center and showed how to calculate it for a trailer. This was followed by the presentation of project goal \#1, to verify that the force and moment arm vectors are perpendicular to each other

The second series of slides presented the concept of finding the force vector applied by the vehicle at the trailer hitch based on its instantaneous center.

The next series of slides focused on the development of the rotational matrix for coordinate transformation. The students were reminded of the use the dot product to calculate vector components, and the rotational matrix was derived based on vector components. An example was presented to further illustrate the use of the rotational matrix to transform a problem from a reference coordinate system to a local coordinate system. Figure 7 is a sample of one of the slides presented in this section.

$$
\begin{aligned}
& \underbrace{\underbrace{\sum_{x, y}}_{\theta}}_{\theta} x \\
& F_{u}=\vec{F}_{x y} \cdot \hat{i}_{u} \\
& \text { special unit vector, }(1,0,0) \\
& \text { based on }(u, v) \text { coords } \\
& F_{u}=\left(F_{x} \hat{i}_{x}+F_{y} \hat{j}_{y}\right) \cdot \hat{i}_{u} \\
& F_{v}=\left(F_{x} \hat{i}_{x}+F_{y} \hat{j}_{y}\right) \cdot \hat{j}
\end{aligned}
$$

Fig. 7. Vector Component and Rotational Matrix Learning Material Example

The final series of slides presented an overview of the analysis required to meet project goal \#2, to develop model predictions for the path of the trailer wheels and verify using the $\mathrm{RC}$ car and trailer prototype. This included an overview of the steps in the mathematical model and a strong encouragement to complete and fully understand the analysis before proceeding to the verification exercise.

\section{RESULTS / SAMPLES OF STUDENT WORK}

The measurements and analysis were conducted by the students over a 3 week period, from October 31 to November 18, 2016. The total class size was 120 students, divided into approximately 30 groups. Each group required approximately 2 hours to complete both 
parts of the data collection exercise, and each group handed in a final report summarizing their findings and conclusions. The following excerpts from a few of these reports are indicative of the results for the class.

\subsection{Force measurement}

Figures $8-10$ shows the results of force measurements for the first part of the exercise, backing up a trailer by hand. From a qualitative point of view the measurements are consistent with expectations; the highest force is measured when the component of the force vector aligned with the direction of travel of the trailer was the largest. The magnitude of the force was higher for an angle of $150^{\circ}$ than $120^{\circ}$, and when the angle approached $90^{\circ}$ the magnitude of the force vector approached zero.

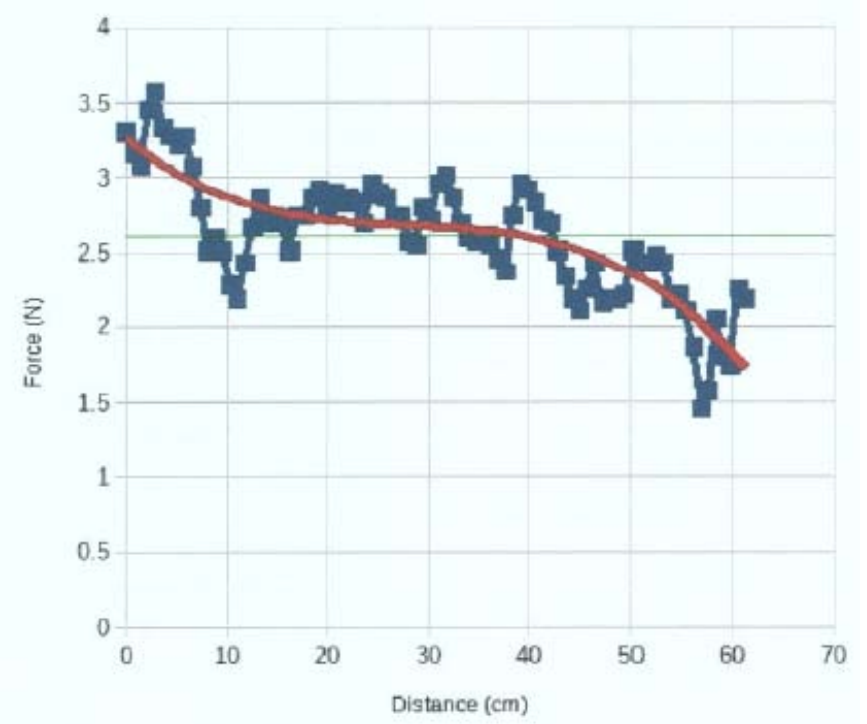

Fig. 8. Force versus Distance, $\theta=150^{\circ}[3]$

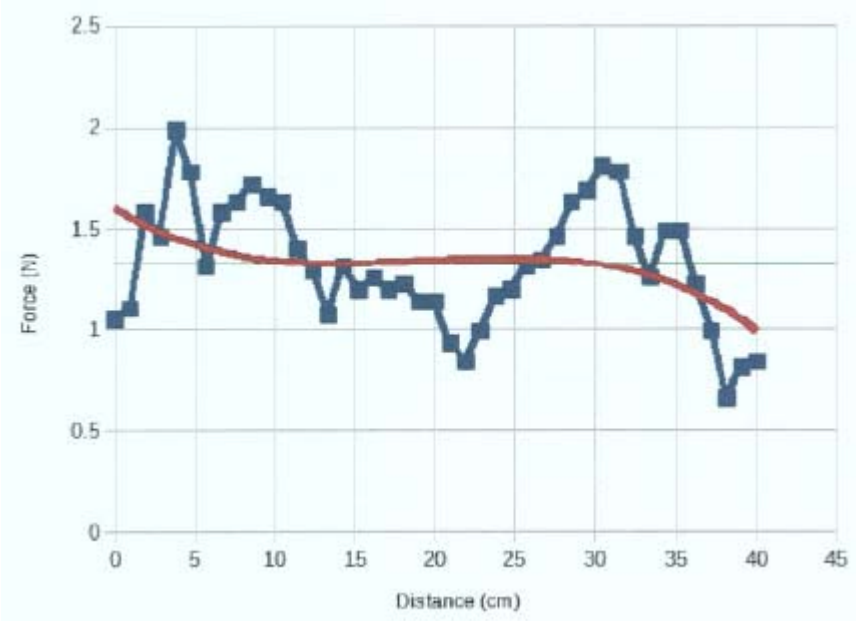

Fig. 9. Force versus Distance, $\theta=120^{\circ}[3]$

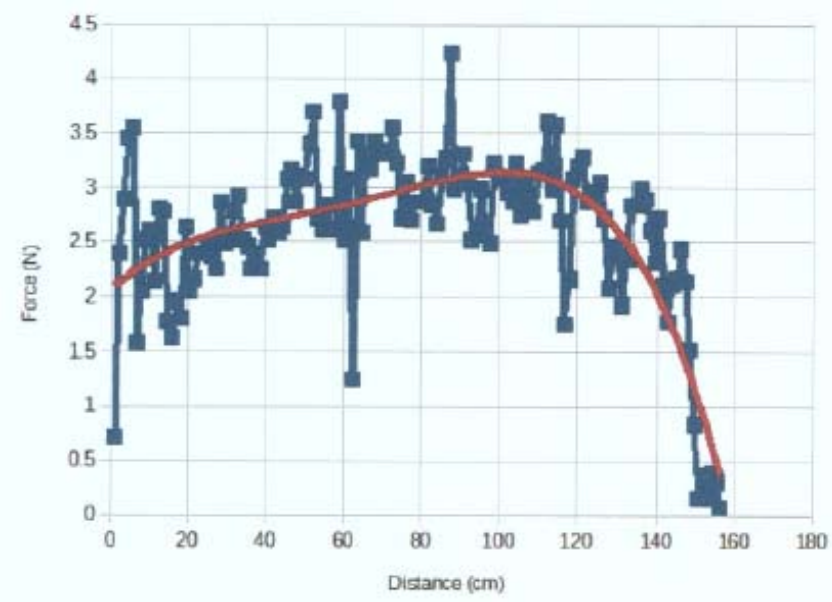

Fig. 10. Force versus Distance, Straight Path [3]

All the data presented by the students showed significant variations, due to inconsistences in how hard and how fast the trailer was pushed. In certain cases these inconsistences were significant enough to mask the expected change in magnitude, leading to confusion among the students as they attempted to interpret the results.

\subsection{Trailer Instantaneous Center.}

Figure 11 shows a schematic and photo presented by one of the student groups of the instantaneous center and the corresponding circular path followed by the wheels for the first part of the exercise, backing up the trailer by hand.

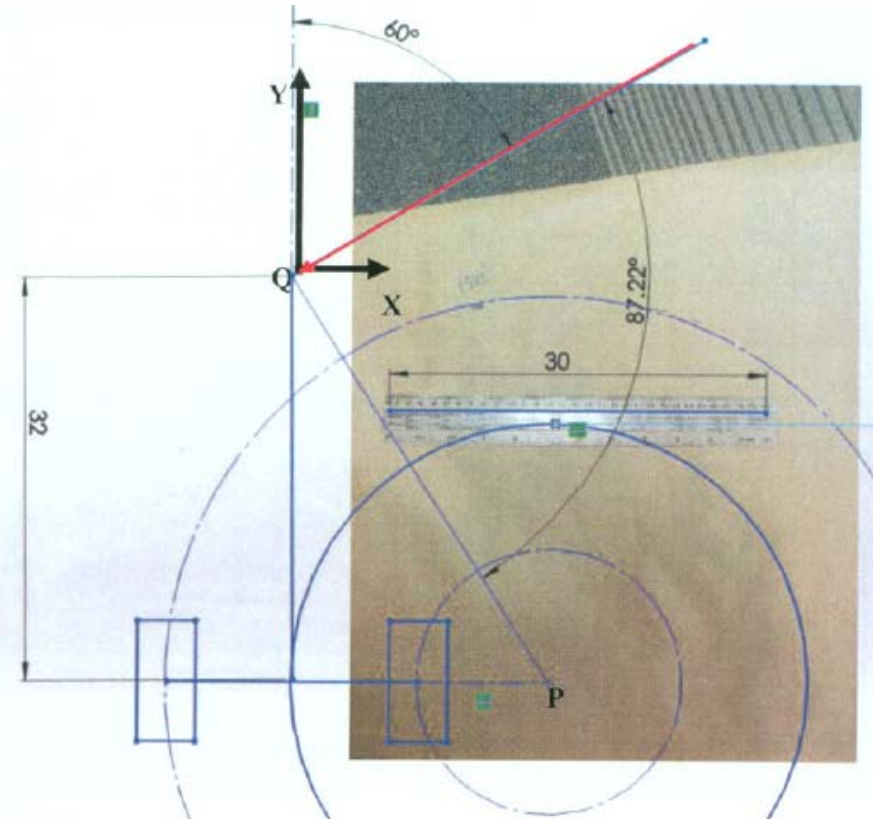


Fig. 11. Instantaneous Center for Part 1 Measurements,

$$
\theta=120^{\circ}[2]
$$

In most cases the students were successful in verifying the normal relationship between the force vector and a vector formed between the instantaneous center and the hitch point. There were a number of groups that seemed to struggle more with this part of the measurements than the second part, and a number of groups booked additional times in the lab to repeat their measurements.

\subsection{Vehicle and Trailer Instantaneous Center}

The most successful part of the project was the verification of the analyzed path of the trailer wheels using the $\mathrm{RC}$ car and trailer prototype. Most groups were able to complete the required analysis for the location and value of the instantaneous center location for the trailer for each of the five test cases successfully on the first attempt. Some groups who had incorrectly predicted the location were quick to identify their error during the verification process. The most common error was in locating the instantaneous center on the wrong side of the trailer.

Figures 12 - 15 show photographs from the student reports comparing the predicted with observed paths followed by the trailer wheels for Cases 1, 2, 4 and 5. In all cases the predicted and observed paths are similar to each other, but in all cases the trailer veers off once the angle at the hitch begins to change significantly.

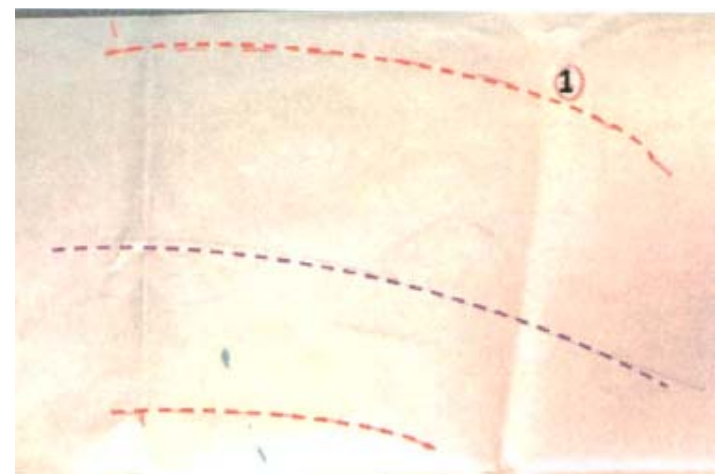

Fig. 12. Predicted (Center Line) versus Observed (Outside Lines) Path of Trailer Wheels for Part 2, Case 1 [2]

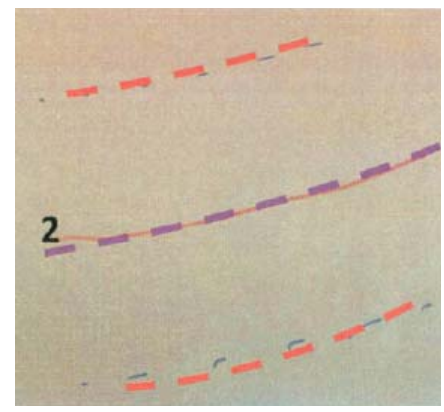

Fig. 13. Predicted (Center Line) versus Observed (Outside Lines) Path of Trailer Wheels for Part 2, Case 2 [2]

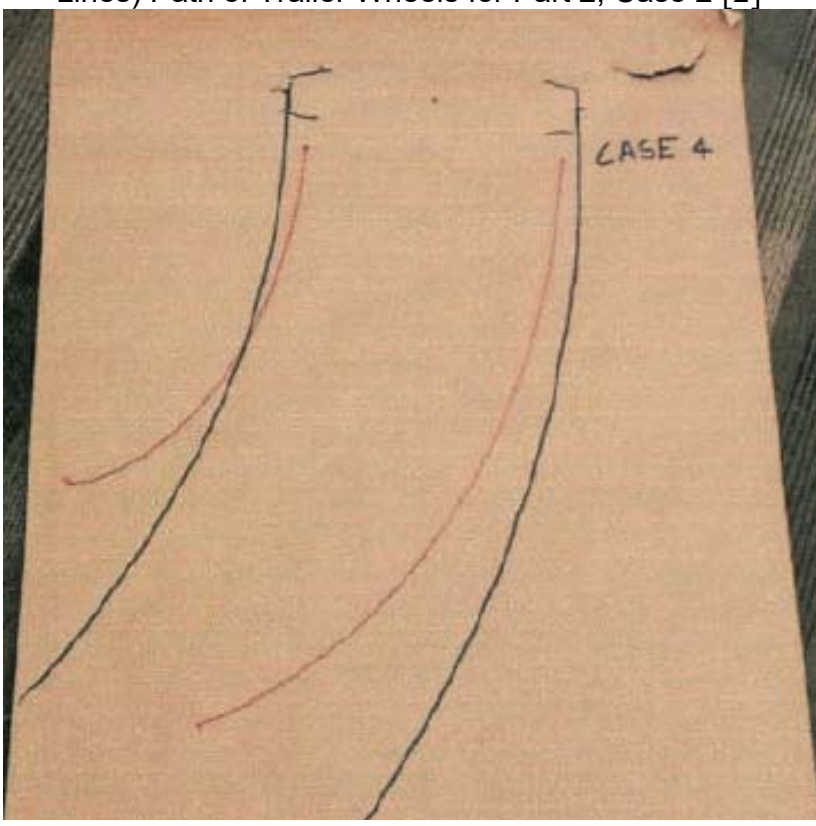

Fig. 14. Predicted (Longer) versus Observed (Shorter) Path of Trailer Wheels for Part 2, Case 4 [3]

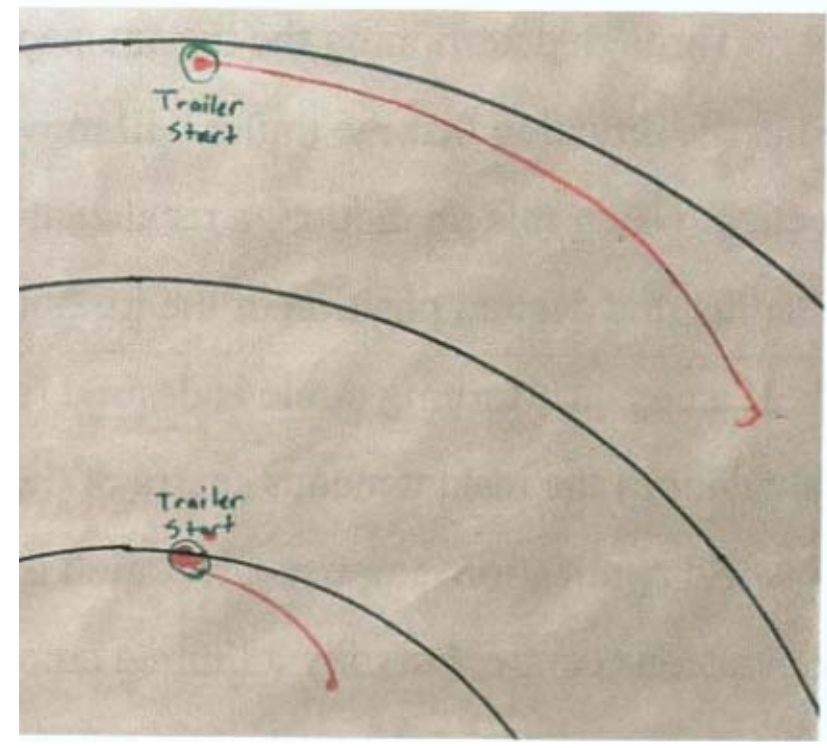

Fig. 15. Predicted (Longer) versus Observed (Shorter) Path of Trailer Wheels for Part 2, Case 5 [4]

\section{SUMMARY AND RECOMMENDATIONS}

An application-based project for a second year Mechanical Engineering calculus course that demonstrates the use of vector products and vector components to predict the path followed by a trailer relative to a vehicle as it is backed up has been presented. The project was successfully implemented in the Fall, 
2016 academic term for a class of approximately 120 students. The project was well received by the students, and most groups were very successful in meeting all the goals of the project and producing an excellent summary report.

After the first implementation of this project, a few key conclusions and recommendations can be made as follows.

Dividing the measurement tasks into two distinct activities, separated by analysis, was highly effective. Students who seemed to struggle with understanding the physical concepts in their first visit to the lab had a much better understanding of the problem in their return visit. This demonstrates the value of early introduction of a hands-on exploration-based activity that gives students a physical understanding of key elements of the problem before they begin the analysis

The data from the force measurements conducted in the first part of the project were difficult to interpret and for many students did not provide a clear picture of the physical nature of vector components. In future implementation is would be helpful to provide a constant force (perhaps use the vehicle and its motor) at a fixed hitch angle.

A stretch goal, to design a control system for backing up a trailer using a vehicle, was too vague and openended for 2A Mechanical Engineering students. Future discussion questions should provide more context to the students.

\section{Acknowledgements}

The financial support of this work by the National Sciences Engineering Research through the NSERC Chair, Engineering IDEAs Clinic is gratefully acknowledged.

\section{References}

[1] Jeffray Behr, Andy Joshi, Brian Mao and Sebastien Wilson, Vector Analysis Project, ME201 Advanced Calculus, Department of Mechanical and Mechatronics Engineering, University of Waterloo, November 24, 2016.

[2] Alyson Colpitts, Janelle Tan, Martin Jaimes, and Solomon Jeyasam, Vector Analysis Project: Backing up a Trailer, ME201 Advanced Calculus, Department of Mechanical and Mechatronics Engineering, University of Waterloo, November 24, 2016.

[3] Alexander Derus, Alexander Forsyth, Andrew Dawson, and Gregory Sinclair, Vector Analysis, ME201 Advanced Calculus, Department of Mechanical and Mechatronics Engineering, University of Waterloo, November 24, 2016.
[4] Adam Marchand, Matthew Hassenbach, Dominik Postl, and Roberto Ferro, Vector Analysis Project: Backing up a Trailer, ME201 Advanced Calculus, Department of Mechanical and Mechatronics Engineering, University of Waterloo, November 17, 2016. 